\title{
A Meniscus Fingering Instability in Viscoelastic Fluids
}

\author{
Baudouin Saintyves, ${ }^{1,2}$ Serge Mora ${ }^{3}$ and Elisabeth Bouchaud ${ }^{2,4}$ \\ ${ }^{1}$ Department of Mechanical Engineering, Massachusetts Institute of Technology - Cambridge, MA 02139, USA \\ ${ }^{2}$ Université Paris-Saclay, Commissariat à l'Énergie Atomique, Institut Rayonnement Matière de Saclay, \\ Service de Physique de l'Etat Condensé, 91191 Gif-sur-Yvette Cedex, France. \\ ${ }^{3}$ LMGC - UMR 5508 Université de Montpellier and CNRS Place E.Bataillon F-34095 Montpellier Cedex 5, France.* \\ ${ }^{4}$ PSL Research University, Ecole Supérieure de Physique et de Chimie Industrielles de Paris, \\ Unité Mixte de Recherche Gulliver, Institut Pierre-Gilles de Gennes, 8 rue Jean Calvin, 75005 Paris, France.
}

\begin{abstract}
We report experiments where a viscoelastic fingering instability develops at the free interface between air and a model viscoelastic fluid confined in a Hele-Shaw cell. The fluid is symmetrically stretched with constant velocity along two opposite directions, leading to the inflation of a twodimensional air bubble growing from a millimetric centered hole. The instability is observed when the circumferential stretch of the inflating bubble reaches a threshold, that depends on the viscoelastic properties of the fluid through the ratio of the material relaxation time to the time elapsed before the fingers start to develop. The critical stretch of the bulk fingering instability of a stretchable elastic solid [B. Saintyves, O. Dauchot, and E. Bouchaud, Phys. Rev. Lett., 111, 047801 (2013)] is recovered for large values of this ratio.
\end{abstract}

\section{INTRODUCTION}

Although the mechanical response of an elastic solid is intrinsically different from that of a liquid, Rayleigh noticed in 1922[1] that the equations governing the deformation of a Hookean solid or a Newtonian liquid are analogous by replacing shear viscosity with shear modulus and velocity with displacement. This analogy holds only for small elastic deformations since it involves Hooke's Law. However, Rayleigh's idea is also fruitful for large deformations: even if the equations are not mathematically analogous, recent studies have underlined striking similitudes in the deformations of elastic solids and viscous liquids, especially if the shear modulus of the solid is low and/or at small length scales. For instance, the adhesion of small particles deposited on a solid surface mimics the adsorption of particles at a fluid interface [2]; the sharp edges of soft elastic solids are rounded by surface tension $[3,4]$, cylinders placed on a soft solid surface interact with each other, leading to an elastic analog of the Cheerios effect for capillary interactions on a fluid interface [5]. The most spectacular occurrences of this analogy arise through mechanical instabilities. A rope falling onto a solid surface typically forms a series of regular coils, reminiscent of viscous coiling $[6,7]$; soft cylinders can develop Rayleigh-Plateau pearling instabilities $[8,9]$; the free surface of a soft elastic solid facing downwards is unstable with respect to the Rayleigh-Taylor instability [10-12] and wrinkles may appear at the free surface of an elastomer film subjected to an electric field [13], a phenomenon first observed with liquid films [14]. More recently, the free surface of a confined elastic layer subjected to a hydrostatic pressure has been shown to undergo a bulk fingering instability [15] reminiscent of the Saffman-Taylor instability [16]. The same instability

*Electronic address: serge.mora@umontpellier.fr has also been observed in an analogous debonding experiment of the same confined elastic layer [17-19] or in a hemispheric probe tack setting [20]. These analogies might provide a powerful method to better understand morphogenesis processes in which the interplay between viscous and elastic mechanical responses are ubiquitous, for instance in life science, where brain tissues or tumors growth can be described with viscoelastic models [21, 22] and in geophysics, in dikes and diapirs formation in viscoelastic rocks [23, 24].

Pattern formation during tensile deformation of confined viscoelastic layers has also been studied using probe tack tests [25], where poly(dimethylsiloxane) with various degrees of cross-linking was used in order to switch continuously from a viscous liquid to an elastic solid. Two distinct regimes of fingering instabilities were observed: a viscoelastic regime related to the Saffman-Taylor instability and an elastic regime with interfacial crack propagation. In these experiments the two regimes correspond to phenomena related to two distinct mechanisms: a bulk phenomenon related to the viscous fingering instability and an interfacial instability.

In contrast, we focus here on a bulk fingering process, and study the transition from elastic fingering to viscoelastic fingering by using model viscoelastic fluids. We first introduce (Section II) these fluids and the Hele-Shaw cell used in this study. The setup is first tested with Newtonian fluids instead of viscoelastic fluids (Section III): no fingering instability is observed. Section IV is devoted to experiments carried out with viscoelastic fluids. A fingering instability is found to grow in the cell beyond a threshold for the air injection rate, that depends on the rheological properties of the fluid. In addition, the destabilization develops beyond a critical circumferential stretch of the inflating bubble. We demonstrate that this critical stretch depends on the fluid properties through the ratio of the material relaxation time to the time elapsed before the occurrence of the destabilization. We show that for high values of this ratio, the critical 
stretch matches with the threshold of the elastic instability previously evidenced in a highly deformable elastic hydrogel [15]. Finally, the possible mechanisms of the viscoelastic fingering instability are discussed in section $\mathrm{V}$, and a short conclusion is proposed at the end of the paper.

\section{EXPERIMENTAL SYSTEM}

The viscoelastic fluids and the experimental setup are introduced in this section.



FIG. 1: Rheological properties of viscoelastic Fluid 3 (see Table I) performed at $T=23^{\circ} \mathrm{C}$ using a strain controlled rheometer ARES from TA instruments in the cone-plate geometry (40 mm diameter and $1.1 \mathrm{deg}$. cone) . The shear stress $\left(\sigma_{x y}\right)$ and the first normal stress difference $\left(N_{1}\right)$ are measured as a function of the steady shear rate in a shear-rate sweep test. The black lines correspond to the prediction of Upper Convected Maxwell Model for a shear modulus and relaxation time equal to $75.8 \mathrm{~Pa}$ and $0.6 \mathrm{~s}$. Top left inset: scheme of a bridged microemulsion. Bottom right inset: Storage $\left(G^{\prime}\right)$ and loss $\left(G^{\prime \prime}\right)$ moduli measured by oscillatory frequency sweep tests. The black lines comes from the Maxwell model, with the same set of shear modulus and relaxation time as previously used (strain amplitude $=10 \%$ ).

We used a model system made of oil-in-water droplets microemulsion connected to each other by telechelic polymers [26]. The droplets are swollen with decane, stabilized by a cationic surfactant (cetyl-pyridinium chloride) and a cosurfactant (n octanol), dispersed in brine $(0,2 \mathrm{M} \mathrm{NaCl})$. The telechelic polymers are homesynthesized triblock copolymers consisting in watersoluble poly(ethylene oxide) (PEO) block of molecular weight $35000 \mathrm{~g} / \mathrm{mol}$, modified by grafting a $\mathrm{C}_{18} \mathrm{H}_{35}$ aliphatic chain at each extremity of the central PEO chain[27]. The degree of functionalization is larger than $98 \%$ and the polydispersity is very low $(p . i .=1.1)$. Those end chains stick reversibly into the hydrophobic core of the oil droplets as shown in an inset of Fig.1. The adhesion energy of a sticker in oil droplets is moderate $\left(\sim 20 k_{B} T\right)$ so that they randomly escape from time to time and reconnect to any accessible droplet and the topography of the network is permanently renewed, allowing stress relaxation and flow $[28,29]$. Since it is formed from incompressible liquids (mainly water and decane), the system itself is incompressible.

This system behaves as a Maxwell viscoelastic fluid, i.e. a fluid characterized by a shear modulus $G$ with a unique relaxation time $\tau_{0}$ (Fig.1). A Maxwell viscoelastic fluid behaves as a viscous fluid at long timescales (larger than $\tau_{0}$ ), and like an elastic solid at short timescales (shorter than $\left.\tau_{0}\right) . \tau_{0}$ and $G$ can be finely tuned by adjusting volume fraction in oil droplets $(\phi)$ and the number of linking chains (polymers) per droplets $(r)$. The shear modulus $G$ and relaxation time $\tau_{0}$ have been measured by standard linear rheological tests performed with an Ares-RFS controlled-strain rheometer in the cone and plate geometry at $23^{\circ} \mathrm{C}$ (See Table I and bottom inset of Fig.1). In addition to this linear rheological behaviour, these fluids are found to follow in good approximation the Upper Convected Maxwell Model. Consistent with this model[30], the shear stress $\sigma_{x y}$ measured in a steady state increases linearly with the shear rate $\dot{\gamma}\left(\sigma_{x y}=G \tau_{0} \dot{\gamma}\right)$ and the first normal stresses difference does it quadratically $\left(N_{1}=2 G \tau_{0}^{2} \dot{\gamma}^{2}\right)$ (Fig.1). This model ceases to describe the mechanical response of these fluids until a macroscopic fracture appears [31, 32] (for a shear strain up to 100\%; Fig.1). Interestingly, the Upper Convected Maxwell Model reduces for short timescales to the neo-Hookean model, which is the simplest model taking into account large strains (in this model, the strain energy density is simply quadratic with respect to finite strains). The viscoelastic fluids used in our experiments can therefore be described by the Upper Convected Maxwell Model, and by the neo-Hookean model for short timescales. In this study we have carried out experiments with six different Maxwellian viscoelastic fluids whose properties are given in Table I.

\begin{tabular}{|l|c|c|c|c|c|}
\hline & $\phi(\%)$ & $r$ & $\tau_{0}(\mathrm{~s})$ & $G(\mathrm{~Pa})$ & $\eta(\mathrm{Pas})$ \\
\hline \hline Fluid 1 & 3 & 2.5 & 0.41 & 36.8 & 15.1 \\
Fluid 2 & 1.75 & 5 & 0.58 & 35.5 & 20.6 \\
Fluid 3 & 2 & 6 & 0.6 & 75.8 & 45.5 \\
Fluid 4 & 3 & 2 & 0.37 & 21.6 & 7.9 \\
Fluid 5 & 8 & 5 & 1.58 & 500 & 790 \\
Fluid 6 & 1.25 & 6 & 0.66 & 26 & 17.2 \\
\hline
\end{tabular}

TABLE I: Composition and linear rheological properties of the viscoelastic fluids (bridged microemulsions) used in the experiments. $r$ is the number of triblock polymers per microemulsion droplet, and $\phi$ is the volume fraction of the droplets. The shear modulus $G$ and the relaxation time $\tau_{0}$ are obtained by linear oscillatory frequency sweep tests. The zero-shear viscosity $\eta$ is equal to $G \times \tau_{0}$.

The experimental device (Fig.2) is an original design, which consists in a sealed Hele-Shaw cell made of 


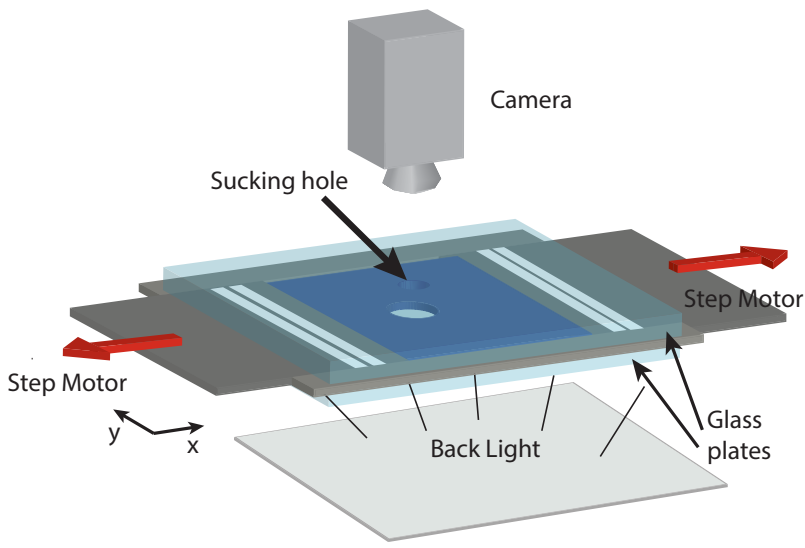

FIG. 2: Experimental set up: a sealed Hele-Shaw cell with two opposite moving walls acting as pistons and pulled at a prescribed velocity $V_{p}$ by synchronized step motors. The gap is fixed to the value $b=2.1 \mathrm{~mm}$.

two $10 \mathrm{~mm}$ thick glass plates of lateral sizes $(250 \mathrm{~mm} \times$ $125 \mathrm{~mm}$ ), with two opposite mobile walls, acting as pistons, and pulled at a prescribed velocity $V_{p}$ by synchronized step motors. The gap is fixed to the value $b=2.1 \mathrm{~mm}$. The pressure decreases in the fluid, induced by the motion of the pistons, sucks air into the filling material. Because our fluids are incompressible, the use of this sucking system allows us to let in air at a constant flow rate which would not be possible with direct blowing. It prevents us to use oil which would have the consequence to waste a lot of material and to have to refill the cell entirely after each experiment. Most observations are top views of the cell recorded from $50 \mathrm{~Hz}$ to $2000 \mathrm{~Hz}$ depending the loading velocity. The protocol used for filling the cell with the viscoelastic fluids is the following: We first fill very slowly the cell at such a speed that the material is far from exhibiting any dominant elastic properties. As the cell is not sealed at this step, the fluid can easily fill the entire space. When this is done, the cell is closed hermetically. Eventually some excess in liquid evacuates from the center hole. We then suck air by pulling out both pistons at $0.01 \mathrm{~mm} \mathrm{~s}^{-1}$ for all the experiment, until the desired initial bubble diameter is reached.

\section{EXPERIMENTS WITH NEWTONIAN FLUIDS}

Investigating first the case of cells filled with Newtonian fluids is a necessary first step, since it represents a benchmark for phenomena occurring in non-Newtonian fluids. This preliminary study dealing with Newtonian fluids is also of fundamental interest: it provides the first comparison between experiments and the old calculation of the shape of a bubble symmetrically stretched in a unidirectional Hele-Shaw cell.

Fig.3(a) shows a time lapse of a moving air/oil interface in an experiment performed with a Newtonian silicon oil. The initial bubble grows first with a quasi-circular shape. Then, it flattens in the vicinity of the fixed walls of the cell, when the distance to the wall becomes smaller than the local radius of curvature of the bubble. This results in an oval and then an oblong shape (Fig.3) the smallest distance of which, separating antipodal points (called below the bubble width) is found to reach a limit value $\lambda W$ at large times, where $W$ is the distance between the two fixed walls of the cell (width of the cell).

The problem of the shape of a developing air-liquid interface in a Hele-Shaw cell has been widely addressed in the context of the viscous fingering instability, e.g. for a gas pushing a viscous liquid in one longitudinal direction of a Hele-Shaw channel [33]. The effects of surface tension on the shape of the finger have been found to be controlled by a unique dimensionless control parameter, the modified capillary number, defined as [34]:

$$
\frac{1}{B}=12\left(\frac{W}{b}\right)^{2} \frac{\eta U}{\sigma},
$$

with $U$ the average velocity of interface, $\sigma$ the surface tension, $\eta$ the viscosity of the fluid, $b$ the thickness of the cell (also called the gap). $1 / B$ is the ratio of the average velocity of the interface to the characteristic velocity produced by the pressure drop across a surface the curvature of which is similar to the width of the cell, $W$. A non zero surface tension is known to have no significant influence on the shape of the fingers arising from the Saffman-Taylor instability provided that $1 / B>1000$ [35]: the fingers are then identical (up to a trivial geometrical scaling factor) whatever the value of $1 / B$. For smaller values of $1 / B$, the relative width $\lambda$ of the fingers increases as $1 / B$ decreases [35].

In our experiments, we measure the apex velocity of the growing bubble $U$, with $V_{p}<U<2 V_{p}$ that we can report in Eq.1, where $V_{p}$ is the velocity of the pistons. In Fig.3$\mathrm{c}$, we have plotted the measured values, $\lambda$, of the relative bubble width at large time, as a function of $1 / B$ for a Newtonian fluid of viscosity $\eta=0.1 \mathrm{Pas}$ (silicon oil, Rhodorsil V100) and for various velocities $V_{P}$. The relative width decreases from $\lambda \simeq 0.7$ to $\lambda=0.48 \pm 0.04$ for increasing values of $1 / B$. Moreover, for $B>1000$ the left and the right sides of the final oblong bubble superimposes with the shape of the Saffman-Taylor finger. A detailed comparison of the relative width of a SaffmanTaylor finger (see the red line in Fig.3(a)) and the final relative width of the bubble measured in our cell highlights quantitatively this outcome. These observations are not surprising since the precise geometry of the air injection is not expected to play a significant role far away, as it is the case in the final stages of the bubble growth. However it provides an argument to state that surface tension effects are negligible compared to viscous effects for $1 / B$ significantly larger than 1000 in our cell. 
Note that the red curve in Fig.3-c overestimates the relative width $\lambda$ for the data point with the smaller value of $1 / B$. This may be the consequence of the finite size of the cell, or a consequence of the finite steps imposed by the stepper motor, that are more pronounced at low speed.

Seguier and Jacquard [36] addressed the problem of a bubble growing from a centred hole in a linear channel of width $W$ initially filled with a viscous Newtonian liquid into a two-dimensional porous medium. The authors have considered symmetric boundary conditions, with purely longitudinal velocities far away from a central hole through which the gas penetrates into the cell:

$$
\left\{\begin{array}{l}
v_{x}= \pm V_{p} \text { and } v_{y}=0 \text { at } \mathrm{x}= \pm \infty \\
v_{y}=0 \text { for } y= \pm W / 2
\end{array}\right.
$$

where $x$ and $y$ are respectively the longitudinal and transverse coordinates of the channel, and $\mathbf{v}=\left(v_{x}, v_{y}\right)$ the fluid velocity.

If surface tension is neglected, the equation for the time evolution of the interface profile $(x, y)$ of the bubble centred at the origin $(x, y)=(0,0)$ was found to be [36]:

$$
\cosh \left(\frac{\pi x}{W}\right)=\exp \left(2 \frac{\pi Q_{s}}{W^{2}} t\right) \cos \left(\frac{\pi y}{W}\right),
$$

where $Q_{s}$ is the surface flow rate, i.e. the increase of bubble surface per unit time. This expression leads at long time to the equation of the a Saffman-Taylor stationary finger for $1 / B \rightarrow \infty$ [37]. The governing equation of Hele-Shaw flows being identical to that of the flow of fluid through a porous medium, Eq.3 is expected to apply to our experiments, in the limit of small surface tension and assuming that the pistons are far away from the bubble interface. In order to compare the observed shape with the predicted one (Eq.3), we determine the surface flow rate by simply measuring the bubble area as function of time (Fig.3(b)). The agreement with Eq.3 is fine except at the final stages, where the shortest distance between the interface and the pistons is not negligible any more with respect to $W$. Note that the surface flow rate measured from the images (Fig.3(b), red line) is slightly smaller than the surface flow rate calculated from the prescribed velocity of the pistons (blue line). This is due to the existence of wetting films left behind the moving interface, the thickness of which depends on the velocity $[39,40]$.

A point which is not addressed by Jacquard and Seguier is the stability of the interface during bubble growth. Experiments carried out with Newtonian fluids (silicon oils) of viscosity $\eta=0.1 \mathrm{~Pa}$ s (Rhodorsil V100, see Fig.3), $\eta=30 \mathrm{Pas}$ (Rhodorsil V30000) and $\eta=100 \mathrm{Pas}$ (Rhodorsil V100000) evidence that the interfaces of the bubbles growing in our setup do not develop any fingering instability, in contrast with the radial fingering instability observed when a less viscous fluid is injected into a more viscous one in a radial Hele-Shaw cell [41].
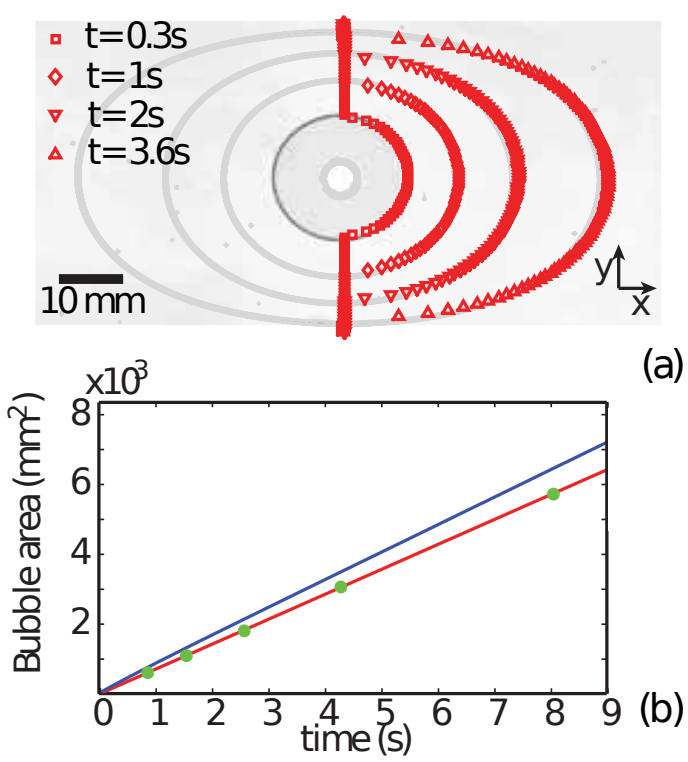

(a)

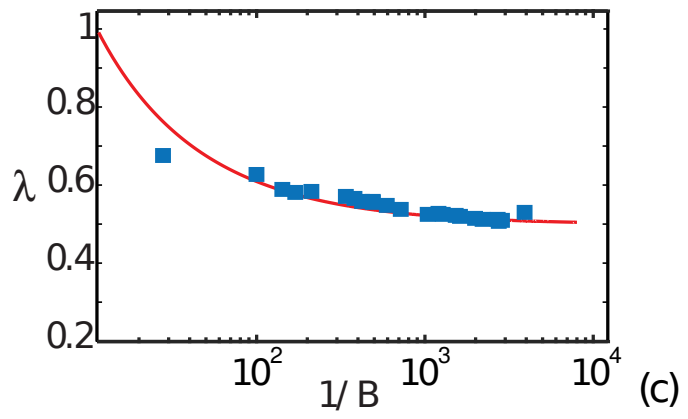

FIG. 3: (a): Comparison between predicted finger profile from Eq.3 and time lapse of an experiment performed with a Newtonian silicon oil (Rhodorsil V100, viscosity $\eta=0.1 \mathrm{Pas}$ ) for a velocity of the pistons $V_{p}=4 \mathrm{~mm} / \mathrm{s}$. (b): Surface flow rate measured experimentally from the picture (red line), and surface flow rate predicted from volume conservation knowing the prescribed velocity of the pistons (blue line). Green disks correspond to this same estimation but with a velocity of the pistons that is directly measured. (c): Relative bubble width. The red curve corresponds to the theoretical prediction from [38].

\section{EXPERIMENTS WITH MODEL VISCOELASTIC FLUIDS}

In the following, we deal with experiments carried out with the viscoelastic Maxwellian fluids. While the interface of the bubbles growing in a Newtonian fluid remains stable, one observes for a wide range of parameters an instability developing at the viscoelastic fluid-air interface. More precisely, when the pistons are pulled out, the bubble is again first quasi-circular. Then three qualitatively distinct behaviors are observed depending on the value of the velocity of the pistons (Fig.4). For the smallest velocities, the evolution of the bubble is similar to that of a Newtonian fluid: the initially quasi-circular bubble elongates (Fig.4(a)) as 




FIG. 4: The various behaviors observed experimentally as a function of the velocity of the pistons (Fluid 2): (a) small velocities; (b) intermediate velocities; (c) high velocities, with fractures.

observed with Newtonian fluids. No interface undulation is observed during the whole process. The slight corrugations that can be observed result from defaults at the cell surface, and they are not amplified during the expansion.

For intermediate velocities of the pistons a destabilization of the quasi-circular interface is observed, leading to the formation of fingers (Fig.4(b)). This observation is in contrast with the previous case and with the stable interface observed with Newtonian fluids of comparable viscosities. At first glance the pattern is similar to a pattern resulting from the radial viscous fingering instability. The destabilization starts for a perimeter of the bubble that depends on the pistons velocity and on the initial size of the bubble.

Finally when the velocity is high enough, the bubble interface first destabilizes with the development of fingers and then, in a second step, an abrupt and quick anisotropic expansion of the air-fluid interface is observed (Fig.4(c)). This sudden process has been observed with the same kind of viscoelastic fluids (bridged microemulsions) in an other Hele-Shaw geometry where it has been shown to correspond to the nucleation and the propagation of fractures in the transient polymer network [32, 42-44]. These fractures developed for a Weissenberg number $\mathcal{W} i$ larger than unity, with $\mathcal{W} i=\frac{\tau_{0} U}{b}$, in good agreement with what is observed in our experiments. Note that the observation of such a sudden event is also reminiscent of fracture observed in polyacrylamide gels [15] in this geometry.

In the following, we focus on the onset of the fingering instability. In order to characterize quantitatively these observations, we measure the parameter

$$
s(t)=\sum_{i>1}\left(a_{i}^{2}+b_{i}^{2}\right) /\left(\bar{R}^{2}+\sum_{i>1}\left(a_{i}^{2}+b_{i}^{2}\right)\right)
$$

where the $a_{i}$ and $b_{i}$ are the Fourier coefficients of the observed pattern and $\bar{R}$ is the mean radius of the bubble. By definition $s(t)$ is always smaller than one. It is equal to 0 if the interface is perfectly circular or ellipsoidal, and increases abruptly as the interface becomes corrugated at time $t_{c}$, yielding a way to precisely determine when the instability starts to develop (Fig.5), and then to calculate the critical circumferential stretch at the onset of fingering defined by $\gamma_{c}=\left(P_{c}-P_{0}\right) / P_{0}$ where $P_{c}$ is the critical interface perimeter corresponding to the first detected undulation, and $P_{0}$ is the initial perimeter (at time 0).

The evolutions of $\gamma_{c}$ with the velocity $V_{p}$ of the pistons for the viscoelastic fluids are plotted in Fig.6 for an initial radius $R_{0}=5.5 \mathrm{~mm}$ of the bubble. At low velocity $\left(V_{P}\right.$ smaller than a value that depends on the rheology of the viscoelastic fluids) no undulation is observable and then $\gamma_{c}$ is not defined. For high enough velocities, undulations appear, as explained above. $\gamma_{c}$ is found to decrease with $V_{P}$ and to reach the plateau value $\gamma_{c}=0.57 \pm 0.07$ for high velocities (Fig.6). Before the plateau value is reached, $\gamma_{c}$ depends both on the piston velocity $V_{p}$ and on the fluid properties (Fig.6). Handling Fluid 5 is a difficult task because of its high viscosity : Only tests at very low speeds or conversely at high speeds are possible because of sealing problems specific to this fluid.

The critical time $t_{c}$ also depends both on the piston velocity $V_{p}$ and on the rheological properties of the fluids. Indeed a straightforward relationship exists between $t_{c}$, $\gamma_{c}$ and $V_{p}$ : As long as the bubble stays almost circular,

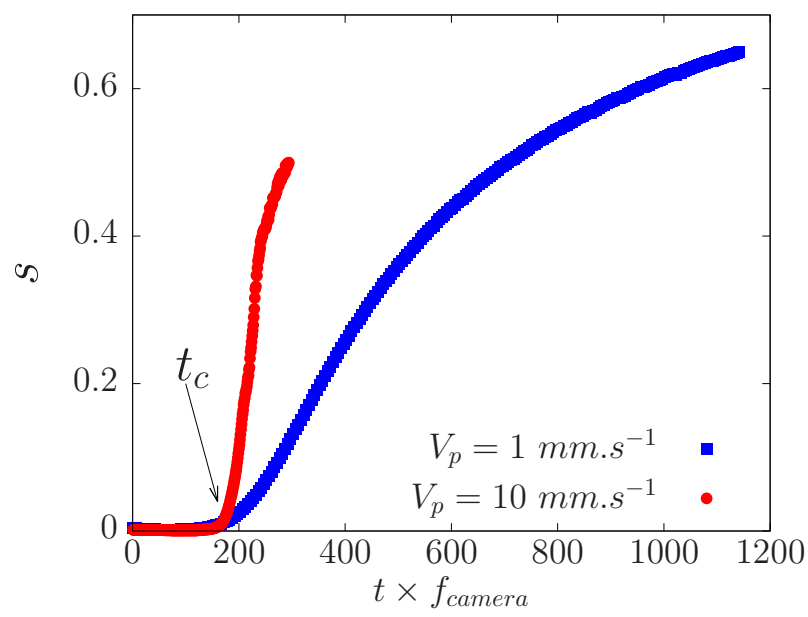

FIG. 5: Value of the quantity $s$ (Fluid 2) as a function of the dimensionless time $t \times f_{\text {camera }}$ where $1 / f_{\text {camera }}$, the inverse of the acquisition frequency, represents the typical experimental time. $f_{\text {camera }}=50 \mathrm{~Hz}$ for the blue curve $\left(V_{p}=1 \mathrm{~mm} \mathrm{~s}^{-1}\right)$ and $f_{\text {camera }}=1500 \mathrm{~Hz}$ for the red curve $\left(V_{p}=10 \mathrm{~mm} \mathrm{~s}^{-1}\right.$; This curve stops when fractures appear). The abrupt increase of $s$ corresponds to the critical time $t_{c}$ at which the interface destabilizes. By measuring the interface perimeter $P$ at this instant, we are able to determine the critical interface deformation $\gamma_{c}=\left(P_{c}-P_{0}\right) / P_{0}$. Similar behaviors are observed with the other tested fluids. 
the fluids being incompressible, its radius $R$ evolves with time $t$ in the following way:

$$
R=\sqrt{R_{0}^{2}+\frac{2 W V_{p} t}{\pi}}
$$

where $R_{0}$ is the initial radius. After a time $t_{c}$, the radius of the bubble has grown from $R_{0}$ to $R_{c}=R_{0}\left(1+\gamma_{c}\right)$, so that (Eq.4) gives

$$
t_{c}=\frac{\pi R_{0}^{2} \gamma_{c}\left(\gamma_{c}+2\right)}{2 W V_{p}} .
$$

Then, although the control parameter in the experiment is $V_{p}$, one can equivalently consider the variation of $\gamma_{c}$ as function of $t_{c}$ in order to analyze the variations of $\gamma_{c}$. Considering in addition the material relaxation time $\tau_{0}$ of the viscoelastic fluids as an other characteristic time of the system, the critical stretch $\gamma_{c}$ is plotted in Fig.7 as function of $\tau_{0} / t_{c}$ for experiments with different fluids but with the same initial radius $\left(R_{0}=5.5 \mathrm{~mm}\right)$. Interestingly, the data points are far less scattered compared to Fig.6, highlighting two different regimes depending on the value of the ratio $\tau_{0} / t_{c}$ : A first regime is observed for $\tau_{0}$ larger than $t_{c}$, where $\gamma_{c}$ as a function of $\tau_{0} / t_{c}$ is almost constant with a similar value for all the viscoelastic fluids $\left(\gamma_{c}=0.57 \pm 0.07\right)$, no matter the value of the shear modulus or the material relaxation time. This plateau value is very close to the measured $\gamma_{c}$ at the threshold of the bulk fingering instability observed previously with the same setup and the same initial diameter but with elastic solids (polyacrylamide gels) of various shear moduli $\left(\gamma_{c}=0.6 \pm 0.04,[15]\right)$. This observation turns out to be true for all the tested initial bubble radii $R_{0}$, as shown in Fig.8, where the value of $\gamma_{c}$ at high velocity is plotted as a function of $b / R_{0}$ for both elastic gels (red circles, from [15]) and viscoelastic fluid (blue squares) and follows $\gamma_{c} \sim 1.75 b / R_{0}$.

The second regime arises at lower $\tau_{0} / t_{c}$, where $\gamma_{c}$ is

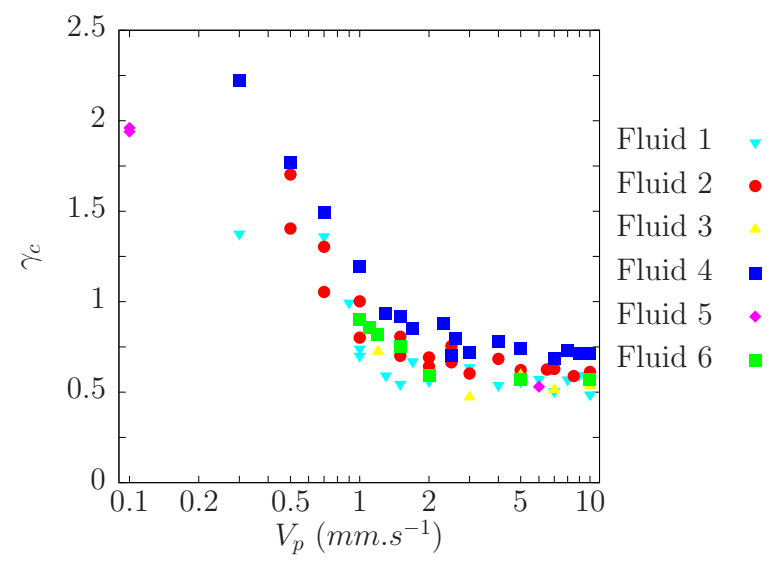

FIG. 6: Critical interface deformation $\gamma_{c}$ (at the onset of the fingering instability) as a function of the velocity of the pistons $V_{p}$ in Log-Lin scales, with $R_{0}=5.5 \mathrm{~mm}$. a decreasing function of $\tau_{0} / t_{c}$. More precisely, as evidenced in Fig.7, $\gamma_{c}$ starts to increase as $\tau_{0} / t_{c}$ decreases for $\tau_{0} / t_{c} \simeq 1$, which is achieved by gradually slowing down the piston velocity from one experiment to another.

\section{DISCUSSION}

The critical circumferential stretch $\gamma_{c}$ is a function of $\tau_{0} / t_{c}$, i.e. the Deborah number [45], for a given value of the geometrical number $R_{0} / b$.

In the limit $\tau_{0} / t_{c} \gg 1, \gamma_{c}$ is similar to the critical stretch of the elastic fingering instability evidenced in [15], whatever the value of $R_{0} / b$. This suggests that in the limit $\tau_{0} / t_{c} \gg 1$, the viscoelastic instability is related to the elastic fingering instability. Indeed, for $t_{c} \ll \tau_{0}$ (the Deborah number is larger than unity), the microscopic configuration of the polymer network of the viscoelastic fluids has not enough time to reorganize during the growth of the bubble, or in other words, the viscoelastic fluid has not flown and exhibits an elastic behaviour. However, all the fingers in the viscoelastic fluids appear simultaneously and grow continuously, in contrast with the purely elastic case [15]. One can think this difference arises from the finite relaxation time of the fluids that tends with the surface tension to smooth the stable interface and to suppress the distributed and frozen defaults that are present in the purely elastic case.

When $\tau_{0} / t_{c}$ decreases, the value of $\gamma_{c}$ continuously increases from the purely elastic value $\left(\gamma_{c} \simeq 0.6\right.$ for $R_{0}=5.5 \mathrm{~mm}$ ) and the observed patterns evolves continuously, showing how the features of the elastic fingering instability are modified when the mechanical response of the sample is gradually changed from elastic to fluid.

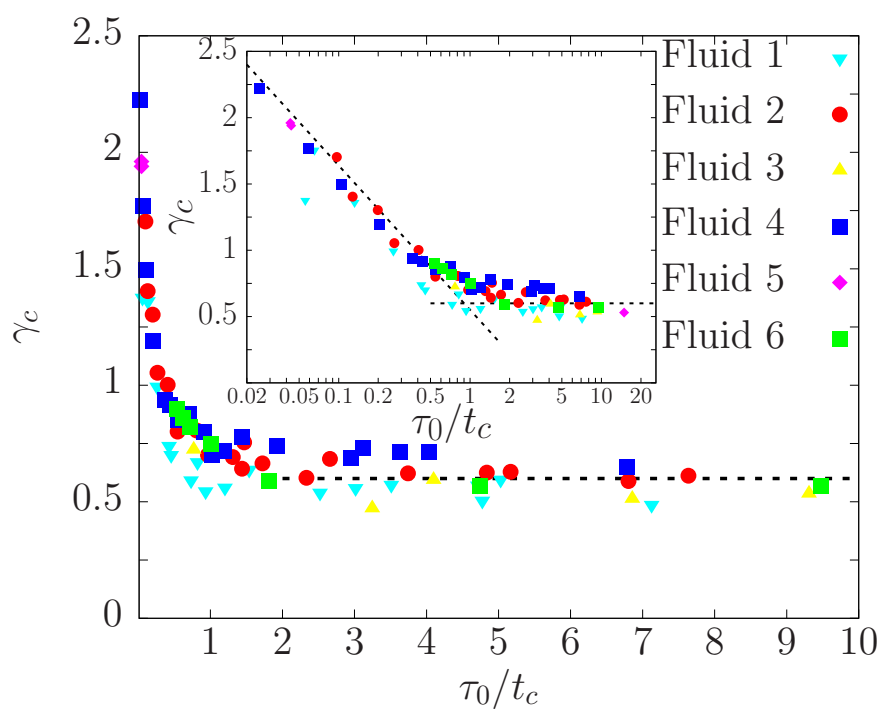

FIG. 7: Critical stretch $\gamma_{c}$ as a function of $\tau_{0} / t_{c}$ for different fluids with $R_{0}=5.5 \mathrm{~mm}$. Inset : same data points in Log-Lin scales. 


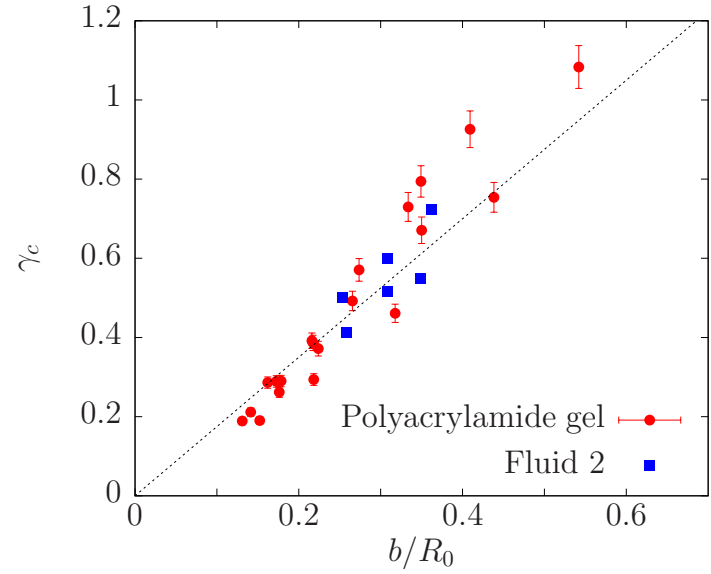

FIG. 8: Critical stretch $\gamma_{c}$ as a function of the geometrical parameter $b / R_{0}$ for the polyacrylamide from [15] and for a bridged microemulsion (Fluid 2).

Note that, since $\tau_{0}=\eta / G$ for Maxwellian viscoelastic fluids, $\tau_{0} / t_{c}$ can be written from Eq.4 as:

$$
\frac{\tau_{0}}{t_{c}} \simeq \frac{\eta 2 W V_{p}}{G \pi\left(R_{c}^{2}-R_{0}^{2}\right)}
$$

Multiplying by $\left(R_{c}-R_{0}\right)^{2} / b^{2}$ both the numerator and the denominator of Eq.6, we obtain an expression for $\tau_{0} / t_{c}$ as an energy ratio:

$$
\frac{\tau_{0}}{t_{c}}=\frac{\frac{\eta}{2} 2 W b V_{p} t_{c}\left(\frac{R_{c}-R_{0}}{b t_{c}}\right)^{2} t_{c}}{\frac{G}{2} \pi\left(R_{c}^{2}-R_{0}^{2}\right) b\left(\frac{R_{c}-R_{0}}{b}\right)^{2}} .
$$

Let us consider two different samples, a Newtonian fluid of viscosity $\eta$ and an elastic solid of shear modulus $G$, and suppose that both sample is placed separately in the cell, the piston velocity being equal to $V_{p}$. The numerator of Eq.7 is the characteristic viscous energy dissipation at time $t_{c}$ in the Newtonian fluid volume $\left(2 W b V_{p} t_{c}\right)$ pushed by the bubble with the average shear rate $\left(R_{c}-R_{0}\right) /\left(b t_{c}\right)$. The denominator represents the strain energy stored in the extra volume created by expanding the bubble in the elastic system submitted to the average shear strain $\left(R_{c}-R_{0}\right) / b$. Hence from Eq.7 one obtains an explicit interpretation of the Deborah number $\tau_{0} / t_{c}$ in terms of viscous energy and strain energy.

Let us go back to the case of viscoelastic fluids of shear modulus $G$ and viscosity $\eta$. We have shown that the elastic regime occurs for $\tau_{0} / t_{c} \gg 1$. From Eq.7, the energy required for the bubble growth in an elastic material of shear modulus $G$ is, in this case, far smaller than the energy required the bubble growth in a Newtonian fluid of viscosity $\eta$. In the viscoelastic regime (for which $\gamma_{c}$ depends on $\tau_{0} / t_{c}$ ) the difference in $\gamma_{c}$ compared to the elastic case is more and more pronounced as $\tau_{0} / t_{c}$ decreases i.e., from Eq.7, as the ratio of the viscous dissipation to the stored strained energy decreases.

The Saffman-Taylor instability is known to occur at the interface between two fluids confined in a Hele-Shaw cell, when the more viscous fluid is pushing the less viscous one. In our cell, we do not observe the SaffmanTaylor instability with Newtonian fluids: we think that the growth rate of the Saffman-Taylor instability is considerably slowed down because of the intrinsic non circularity of the air bubble in the specific geometry of the cell $[33,46]$. The characteristic time of the instability is therefore far larger than $\tau_{0}$. The Deborah number (defined here as $\tau_{0}$ times the growth rate of the Saffman-Taylor instability in this geometry for a Newtonian fluid with the same viscosity and surface tension) is therefore far smaller than unity, an argument to infer that elastic effects in the Saffman-Taylor mechanism are here negligible [42, 47]. It is therefore unlikely that the observed instability simply results from an amplification of the growth rate of a Saffman-Taylor like instability, due to the viscoelastic feature of the fluids. Additional experiments in which air would be replaced by a Newtonian fluid with a viscosity higher than the viscosity of the viscoelastic fluid would be useful to unambiguously support this point.

\section{CONCLUSION}

An instability at the free surface of air bubbles inflating in a Hele-Shaw filled with viscoelastic fluids has been evidenced. Experiments have been carried out with model Maxwellian viscoelastic fluids, characterized by their shear modulus and their relaxation time. Fluids with different shear modulus and relaxation times have been tested. The fingering instability occurs when the circumferential stretch of the bubble exceed a threshold that does not depend on the shear modulus of the fluid, but on its relaxation time, the air bubble growth rate, the initial size of the bubble, and the gap of the Hele-Shaw cell. For large Deborah number, the critical circumferential stretch at the onset of the observed fingering instability is identical to that of the elastic fingering instability. Viscoelastic features appear continuously upon a gradual decrease of the Deborah number. Since most of complex fluids are viscoelastic (but with other non Newtonian features) our conclusions are expected to be relevant for a broad variety of systems, in particular in life science where the typical stiffnesses of materials are comparable to those of our viscoelastic samples (10-1000 Pa).

These results provide clues for a better understanding of complex interfacial morphogenesis processes in nature involving both elastic and viscous forces. For instance, only a few recent models have taken into account mechan- 
ical effects to predict shapes and characteristic length scales in biological tissues[48-50], while growing tissues has been shown to present viscoelastic properties [22]. Furthermore growth under confinement is a common situation in biology, for example in lung [50-52] or in saliva glands [53] growth.

\section{Acknowledgements}

The authors thank V. Padilla and C. Gasquet for their technical support. This work has been supported by
ANR under contract No. ANR-2010-BLAN-0402-1. B.S. and E.B thank D. Bonn and J.-P. Bouchaud for interesting discussions, and $\mathrm{H}$. Montes for allowing them to perform linear rheology measurements with her equipment. S.M. thanks Y. Pomeau for fruitful and stimulating discussions, and T. Phou for the synthesis of the telechelic polymers. The authors also thank a referee for the suggestion of interpreting the Deborah number in terms of energies.
[1] Lord Rayleigh. Theory of Sound, volume II. Dover, 1922.

[2] R.W. Style, C. Hyland, R. Boltyanskiy, J.S. Wettlaufer, and E.R. Dufresne. Surface tension and contact with soft elastic solids. Nature Communications, 4:2728, 2013.

[3] S. Mora, C. Maurini, T. Phou, J. M. Fromental, B. Audoly, and Y. Pomeau. Solid drops: Large capillary deformations of immersed elastic rods. Phys. Rev. Lett., 111:114301, 2013.

[4] S. Mora and Y. Pomeau. Softening of edges of solids by surface tension. Journal of Physics: Cond. Matt., $27: 194112,2015$

[5] A. Chakrabarti, L. Ryan, M. K. Chaudhury, and L. Mahadevan. Elastic cheerios effect: Self-assembly of cylinders on a soft solid. Europhysics Letters, 112:54001, 2015.

[6] N.M. Ribe. Coiling of viscous jets. Proc. Royal Soc. A, 460:3223-3239, 2004.

[7] M. Habibi, N.M. Ribe, and D. Bonn. Coiling of elastic ropes. Phys. Rev. Lett., 99:154302, 2007.

[8] J. Plateau. Statique Expérimentale et théorique des Liquides Soumis aux Seules Forces Moléculaires, volume II. Gauthier Villars, Paris, 1873.

[9] S. Mora, T. Phou, J.M. Fromental, L.M. Pismen, and Y. Pomeau. Capillarity driven instability of a soft solid. Phys. Rev. Lett., 105:214301, 2010.

[10] A. Chakrabarti, S. Mora, F. Richard, T. Phou, J.-M. Fromental, Y. Pomeau, and B. Audoly. Selection of hexagonal buckling patterns by the elastic Rayleigh-Taylor instability. Journal of the Mechanics and Physics of Solids, 121:234-257, 2018.

[11] S. Mora, T. Phou, J. M. Fromental, and Y. Pomeau. Gravity driven instability in solid elastic layers. Phys. Rev. Lett., 113:178301, 2014.

[12] L. J. Rayleigh. Investigation of the character of the equilibrium of an incompressible heavy fluid of variable density. Proc. London. Math. Soc., 14:170-177, 1883.

[13] Q. Wang and X. Zhao. Creasing-wrinkling transition in elastomer films under electric fields. Phys. Rev. E, 88:042403, 2013.

[14] E. Schaffer, T. Thurn-Albrecht, TP. Russel, and U. Steiner. Electrically induced structure formation and pattern transfer. Nature, 403:874, 2000.

[15] B. Saintyves, O. Dauchot, and E. Bouchaud. Bulk elastic fingering instability in hele-shaw cells. Phys. Rev. Lett., 111:047801, 2013.

[16] P.G. Saffman and G. Taylor. The penetration of a fluid into a porous medium or hele-shaw cell containing a more viscous liquid. Proc. of the Royal Soc. of London, Series A, Math. and Phys. Sci., 245:312-329, 1958.

[17] A. Ghatak, M.K. Chaudhury, V. Shenoy, and A. Sharma. Meniscus instability in a thin elastic film. Phys. Rev. Lett., 85:4329, 2000.

[18] J. S. Biggins, B. Saintyves, Z. Wei, E. Bouchaud, and L. Mahadevan. Digital instability of a confined elastic meniscus. PNAS, 110:12545-12548, 2014

[19] S. Lin, Y. Mao, R. Radovitzky, and X. Zhao. Instabilities in confined elastic layers under tension: Fringe, fingering and cavitation. Journal of the Mechanics and Physics of Solids, 106:229-256, 2017.

[20] K.R. Shull, C.M. Flanigan, and A.J. Crosby. Fingering instabilities of confined elastic layers in tension. Phys. Rev. Lett., 84:3057, 2000.

[21] K. Miller and K. Chinzei. Mechanical properties of brain tissue in tension. Journal of Biomechanics, 35(4):483 490, 2002.

[22] D. Gonzalez-Rodriguez, K. Guevorkian, S. Douezan, and F. Brochard-Wyart. Soft matter models of developing tissues and tumors. Science, 338(6109):910-917, 2012.

[23] O. Galland, S. Burchardt, E. Hallot, R. Mourgues, and C. Bulois. Dynamics of dikes versus cone sheets in volcanic systems. Journal of Geophysical Research: Solid Earth, 119(8):6178-6192, 2014.

[24] Allan M. Rubin. Dikes vs. diapirs in viscoelastic rock. Earth and Planetary Science Letters, 117(3):653 - 670, 1993.

[25] J. Nase, Lindner A., and Creton C. Pattern formation during deformation of a confined viscoelastic layer: From a viscous liquid to a soft elastic solid. Phys. Rev. Lett., 101:074503, 2008.

[26] F. Molino, J. Appell, M. Michel, G. Porte, S. Mora, and E. Sunyer. A transient network of telechelic polymers and microspheres: structure and rheology. J. Phys.: Condens Matter, 12:A491-A498, 2000.

[27] C. Rufier, A. Collet, M. Viguir, J. Oberdisse, and S. Mora. Asymmetric end-capped poly(ethylene oxide). synthesis and rheological behavior in aqueous solution. Macromolecules, 41:5854-5862, 2008.

[28] F. Tanaka and S.F. Edwards. Viscoelastic properties of physically cross-linked networks - transient network theory. Macromolecules, 25:1516-1523, 1992.

[29] M.S. Green and A.V. Tobolsky. A new approach to the theory of relaxing polymeric media. J. Chem. Phys., 14:80, 1946 
[30] C.W. Macosko. Rheology : principles, measurements and applications. Wiley-VCH, New York, 1994.

[31] H. Tabuteau, S. Mora, G. Porte, M. Abkarian, and C. Ligoure. Microscopic mechanisms of the brittleness of viscoelastic fluids. Phys. Rev. Lett., 102:155501-155504, 2009.

[32] C. Ligoure and S. Mora. Fractures in complex fluids: the case of transient networks. Rheologica Acta, pages 91-114, 2013.

[33] D. Bensimon, L.P. Kadanoff, S. Liang, B.I. Shraiman, and C. Tang. Viscous flows in two dimensions. Rev. Mod. Phys., 58:977, 1986.

[34] G.M. Homsy. Viscous fingering in porous media. Annual Review of Fluid Mechanics, 19:271-311, 1987.

[35] J.W. MC Lean and P.G. Saffman. The effect of surfacetension on the shape of fingers in a hele-shaw cell. Journal of Fluid Mechanics, 102:455-469, 1981.

[36] P. Jacquard and P. Seguier. Mouvement de deux fluides en contact dans un milieu poreux. J. Mec, 1:367-394, 1962.

[37] S.D. Howison. Fingering in hele-shaw cells. J. Fluid. Mech., 167:439-353, 1986.

[38] R. Combescot, T. Dombre, V. Hakim, Y. Pomeau, and Pumir A. Shape selection of saffman-taylor fingers. Phys. Rev. Lett., 56:2036-2039, 1986.

[39] F. P. Bretherton. The motion of long bubbles in tubes. Journal of Fluid Mechanics, 10(2):166-188, 1961.

[40] P. Tabeling and A. Libchaber. Film draining and the saffman-taylor problem. Phys. Rev. A, Rapid Com., 33:794-796, 1986.

[41] J. A. Miranda and M. Widom. Radial fingering in a hele-shaw cell: a weakly nonlinear analysis. Physica D, 120:315-328, 1998.

[42] S. Mora and M. Manna. Saffman-taylor instability of viscoelastic fluids: From viscous fingering to elastic fractures. Physical Review E, 81(2):026305, 2010.

[43] G. Foyart, L. Ramos, S. Mora, and C. Ligoure. The fingering to fracturing transition in a transient gel. Soft Matter, 9:7775-7779, 2013.

[44] G. Foyart, C. Ligoure, S. Mora, and L. Ramos. Rearrangement zone around a crack tip in a double selfassembled transient network. ACS Macro Lett., 5:10801083, 2016.

[45] M. Reiner. The deborah number. Physics Today, 17:62, 1964.

[46] D. Bensimon, P. Pelce, and B.I. Shraiman. Dynamics of curved fronts and pattern selection. Journal de Physique, 48:2081-2087, 1987.

[47] S. Mora and M. Manna. From viscous fingering to elastic instabilities. Journal of Non-Newtonian Fluid Mechanics, pages 30-39, 2012.

[48] T. Savin, N. A. Kurpios, A. E. Shyer, P. Florescu, H. Y. Liang, L. Mahadevan, and C. J. Tabin. On the growth and form of the gut. Nature, 476(7358):57-62, August 2011.

[49] T. Tallinen, J.Y. Chung, F. Rousseau, N. Girard, J. Lefevre, and L. Mahadevan. On the growth and form of cortical convolutions. Nat Phys, 12:588-593, February 2016.

[50] V.D. Varner, J.P. Gleghorn, E. Miller, D.C. Radisky, and C.M. Nelson. Mechanically patterning the embryonic airway epithelium. Proceedings of the National Academy of Sciences, 112(30):9230-9235, 2015.

[51] RJ. Metzger, OD. Klein, GR Martin, and MA. Krasnow. The branching programme of mouse lung development. Nature, 453:745, 2008.

[52] R. Clement, P. Blanc, B. Mauroy, V. Sapin, and S. Douady. Shape self-regulation in early lung morphogenesis. PLUS ONE, 7:e36925, 2012.

[53] J. Harunaga, J.C. Hsu, and K.M. Yamada. Dynamics of salivary gland morphogenesis. Journal of Dental Research, 90(9):1070-1077, March 2011. 